\title{
Aeroelastic Analysis of SUGAR Truss-Braced Wing Wind-Tunnel Model Using FUN3D and a Nonlinear Structural Model
}

\author{
Robert E. Bartels* \\ Robert C. Scott ${ }^{\dagger}$ \\ NASA Langley Research Center, Hampton, VA \\ Timothy J. Allen \\ Boeing Research and Technology, Huntington Beach, CA \\ Bradley W. Sexton ${ }^{\S}$ \\ Boeing Research and Technology, St. Louis, MO
}

\begin{abstract}
Considerable attention has been given in recent years to the design of highly flexible aircraft. The results of numerous studies demonstrate the significant performance benefits of strut-braced wing (SBW) and trussbraced wing (TBW) configurations. Critical aspects of the TBW configuration are its larger aspect ratio, wing span and thinner wings. These aspects increase the importance of considering fluid/structure and control system coupling. This paper presents high-fidelity Navier-Stokes simulations of the dynamic response of the flexible Boeing Subsonic Ultra Green Aircraft Research (SUGAR) truss-braced wing wind-tunnel model. The latest version of the SUGAR TBW finite element model (FEM), v.20, is used in the present simulations. Limit cycle oscillations (LCOs) of the TBW wing/strut/nacelle are simulated at angle-of-attack (AoA) values of $-1,0$ and +1 degree. The modal data derived from nonlinear static aeroelastic MSC.Nastran solutions are used at AoAs of -1 and +1 degrees. The LCO amplitude is observed to be dependent on AoA. LCO amplitudes at -1 degree are larger than those at +1 degree. The LCO amplitude at zero degrees is larger than either -1 or +1 degrees. These results correlate well with both wind-tunnel data and the behavior observed in previous studies using linear aerodynamics. The LCO onset at zero degrees AoA has also been computed using unloaded v.20 FEM modes. While the v.20 model increases the dynamic pressure at which LCO onset is observed, it is found that the LCO onset at and above Mach 0.82 is much different than that produced by an earlier version of the FEM, v. 19.
\end{abstract}

\section{Introduction}

Considerable attention has been given in recent years to the design of highly flexible aircraft. The use of modern structural optimization methods, combined with new materials and manufacturing techniques, has enabled aircraft designers to minimize weight while maintaining the required wing strength but with overall increased wing flexibility. The reduction of structural weight has obvious advantages to efficiency, but the result is the necessity of a simultaneous consideration of aerodynamics, structures and possibly control systems in the design of such a flexible vehicle. Such a coupling of methods allows the designer to take advantage of wing shape in achieving optimal aerodynamics. Besides the advantages that are opened up by a very flexible vehicle, such as active wing shaping for minimizing drag, potential aeroelastic issues also arise, such as excessive gust loading, unacceptable flutter margin, and destablizing aero-servoelastic coupling. The result of these advantages and issues is that a multidisciplinary analysis and optimization of the vehicle aerodynamics, structure and control system is likely to be required earlier in the design.

Many optimization studies have used linear aerodynamic theory combined with linear and possibly nonlinear structural models, although recently there has been an exploration of structural optimization using steady and unsteady high-fidelity computational fluid dynamics (CFD).${ }^{1,2}$ Nonconventional vehicle configurations have begun to receive

*Senior Aerospace Engineer, Aeroelasticity Branch, Senior Member.

${ }^{\dagger}$ Senior Aerospace Engineer, Aeroelasticity Branch, Associate Fellow.

\$Principle Engineer.

$\S$ Principle Engineer, Associate Fellow. 
attention in aircraft design optimation studies. Research groups, such as the Virginia Tech Multidisciplinary Aircraft Design group, or that at Georgia Tech, have been developing optimization tools capable of analyzing strut-braced wing (SBW) and truss-braced wing (TBW) designs. ${ }^{4,5,6,8}$ The results of numerous studies demonstrate the significant performance benefits of the SBW and TBW configurations for both long and medium range missions. . $^{3,9,7}$

The aerodynamic aspects of the TBW configuration that advance these metrics are its larger aspect ratio, wing span and thinner wings. The increase in aspect ratio results in a reduction of induced drag, while the reduction in transonic wave drag and parasite drag, because of a thinner wing, allows the wing design to unsweep. This results in laminar flow over a greater portion of the wing. However, from an aerodynamic performance stand point, the strut also has interference drag at wing/strut or wing/jury intersections, which needs to be considered. The structural advantages of the SBW vehicle is its superior load bearing potential. In addition, it has been found that adding a single jury to a strut-braced wing configuration results in a further reduction of wing weight. ${ }^{9}$ The multiple load paths of a strut and jury (truss-braced wing) reduce the bending moment supported by the inner wing and allow an inboard wing box to be lighter, thinner and thus more flexible. It also results in wing, strut and jury members that are in tension or compression, rather than bending only. This can have a significant impact on the aeroelastic behavior of the vehicle. Other studies have shown that the critical structural failure case for the single strut is buckling at $-1 \mathrm{~g}$ loading. ${ }^{3}$ It has also been observed that some TBW designs can result in a flutter weight penalty, possibly requiring active suppression, to relieve flutter onset. ${ }^{7}$ All these factors influence which analyses will be required for the design of a TBW aircraft, and of present interest, the nature of the modeling required for the analysis of flutter.

The typical design Mach number of these studies is in the high subsonic range. The use of linear aerodynamics, corrected with CFD results, may be sufficient for an early design optimization. ${ }^{10}$ At some point, higher fidelity aeroelastic tools will be needed to validate the design. The complexity of a wing/strut configuration suggests the use of higher fidelity tools to simulate the aerodynamic/structure interaction.

The NASA Fixed Wing program recently funded the Boeing Company to perform a design optimization of a TBW. ${ }^{11}$ The goal was the development of an aircraft configuration that advances fuel efficiency and enables noise reduction. The Boeing Company is just now completing an $\mathrm{N}+3$ phase 2 study of a TBW concept. ${ }^{11}$ This study included an analysis and design of a flight vehicle, and the design and testing of an aeroelastic model in the NASA Langley Research Center Transonic Dynamics Tunnel (TDT). ${ }^{12}$ The Boeing designed Subsonic Ultra Green Aircraft Research (SUGAR) TBW wind-tunnel model was tested at the TDT in the December 2013-April 2014 time frame. A photograph of the SUGAR TBW model in the TDT is shown in Figure 1. The purpose of that test was to assess the open- and closed-loop aeroelastic stability of the SUGAR TBW model in an unperturbed or an oscillating free stream. Recently, flutter onset using linear aerodynamics and nonlinear static aeroelastic solutions at various AoAs has been presented using the SUGAR TBW model. ${ }^{10}$

Reference 13 compared aeroelastic LCO results with experimental data from the TDT test. In that study, unsteady simulations were performed in which CFD was coupled with the structural modes of the TBW wind-tunnel model. LCO was observed above an onset boundary that involved wing bending and torsion. Only zero degree AoA conditions were considered, thus allowing the use of structural modes from an unloaded vehicle. By considering an essentially unloaded vehicle, structural nonlinearity could be neglected. This paper builds on that work to include high-fidelity aeroelastic solutions using normal modes evaluated about nonlinear static aeroelastic solutions, using the latest version of the FEM. The focus will be on simulations performed with linear and nonlinear aerodynamics using the nonlinear structural model. After discussing the structure and aerodynamic models, the transfer process between the computational structural dynamics (CSD) and CFD models will be discussed. The paper will conclude with a discussion of results.

\section{Structural Model}

As part of the aeroelastic analysis of the wind-tunnel model, twenty versions of the semi-span FEM were developed. The last two versions of the FEM, version 19 and 20, have approximately 400 nodes with 200 elements including CBEAM, CONM2, RBAR, and RBE2 element types. These models consist of beams for the fuselage, wing, strut, engine nacelle pylon and control surface hinges. Discrete weights are included to account for the control surface actuators, the strut/wing and the engine nacelle pylon/wing attachments. All of these elements are connected to the beams by flexible and rigid body elements. The version 19 FEM (v.19) includes before-test updates to correlate with in-tunnel ground vibration test (GVT) data. This version was used to design the control laws for flutter suppression. Mode shapes and frequencies from v. 19 were also used by Bartels et al. ${ }^{13}$ to generate high-fidelity CFD flutter/LCO simulations.

V.20 of the SUGAR TBW FEM was developed after the wind-tunnel test. This model is shown in Figure 2a. 
It was created by updating stiffnesses to match the post-test GVT data. Correlation of mode 3 with GVT data was improved by decreasing bending stiffness on the strut attachment beam and on certain wing elements. Bending stiffness on certain wing elements was increased in order to improve the correlation of modes 1 and 5 with the GVT data. Correlation of mode 4 with GVT data was improved by adjusting torsional stiffness on the inner wing elements. ${ }^{12}$ The resulting modal frequences are illustrated in Figure 3, where the frequencies of the first five modes for the v.19 and v.20 SUGAR TBW FEMs are shown. Previous studies have shown that flutter or LCO occurs as a result of a coalescence of the $2^{\text {nd }}$ out-of-plane wing bending ( $3^{\text {rd }}$ mode) and the $1^{\text {st }}$ wing/nacelle torsion $\left(4^{\text {th }}\right.$ mode) ${ }^{10,13}$ It is important to note that the frequency of the $3^{r d}$ mode in v.20 is significantly lower than the corresponding v.19 frequency. This difference has a notable influence on flutter/LCO onset for the SUGAR TBW model.

Static and dynamic aeroelastic analyses can be performed with a structural model that is either linear without prestress, linear, or nonlinear with pre-stress. In this work, dynamic aeroelastic analyses are performed with a structural model that is linear, using normal modes. However, the normal modes may be evaluated about a model that is unloaded or one that includes pre-stress and nonlinearity. For cases at zero degree AoA, normal modes for an unloaded model are used. At a non-zero AoA, pre-stressed modes are used from a nonlinear static aeroelastic solution. The structural dynamics equations that include geometric nonlinearity are

$$
\mathbf{M u ̈}+\mathbf{C u}+\mathbf{K}(\mathbf{u}) \mathbf{u}=\mathbf{f}
$$

where $\mathbf{u}=(u, v, w)$ are displacements and $\mathbf{f}$ are the aerodynamic load. This system is approximated using the transformation $\eta=\Phi \mathbf{u}$. The matrix $\Phi$ contains the vibration modes derived about the nonlinear static equilibrium solution $\mathbf{u}_{\mathbf{s}}$ and system parameters $\mathbf{M}, \mathbf{K}\left(\mathbf{u}_{\mathbf{s}}\right)$. The nonlinear static aeroelastic solution has external forcing, $\mathbf{f}$, that includes the aerodynamic forcing and the $1 g$ wing/strut/nacelle weight. In all cases, the structural damping has been estimated from GVT data to be around one percent. The MSC.Nastran nonlinear static aeroelastic solution process is shown in Figure 4.

Nonlinear static aeroelastic solutions using the v.20 FEM have been obtained at Mach numbers 0.70, 0.75, 0.78 and 0.82 and AoA of -1 and +1 degree. Mode shapes and frequencies have been obtained from these solutions. Table 1 shows the frequencies of the first five modes at Mach 0.75 and dynamic pressure of 100 psf and AoA of -1 and +1 degree. For reference, the frequencies for an unloaded vehicle are also shown.

\section{Aerodynamic Modeling}

Linear vortex-lattice or doublet-lattice theory is used for the low-order aerodynamic modeling. Figure $2 \mathrm{~b}$ shows the aerodynamic panel layout used for the nonlinear static and linear flutter analyses. The aerodynamic loads from the panels are transferred to the structural model using a combination of infinite plate and infinite beam splines. There are four major spline regions for the four major aero groups seen in Figure 2b: engine/nacelle, wing, strut, and fuselage. Also, each control surface has spline points associated with them as well.

The high-fidelity simulations are performed by the Navier-Stokes code FUN3D (fully unstructured Navier-Stokes three-dimensional) version 12.6. ${ }^{14}$ The compressible Reynolds-averaged Navier-Stokes (RANS) flow equations and the Spalart-Allmaras turbulence model are solved on a tetrahedral grid. The SUGAR TBW mesh used in the FUN3D calculations is identical to that used and discussed in more detail in Reference 13. A semi-infinite tetrahedral volume grid having 4.5 million nodes was developed with the VGRID software. ${ }^{15}$ The wind-tunnel wall is treated as a symmetry plane. The surface mesh with symmetry plane are shown in Figure 5.

\section{Mode Shape Transfer Between Dissimilar CSD/CFD Models}

Deflection and force transfer between dissimilar CSD and CFD meshes require treatment. Both the MSC.Nastran flutter and the CFD aeroelastic simulations use a modal representation of the structure. In light of the structural model described earlier, the transfer of modal deflections is between a complex interconnection of multiply oriented beams and the aerodynamic surface nodes. The method to transfer node deflections from the beam model to the CFD surface mesh is a simplification of that discussed in Reference 13. For this work, several intermediate steps and an intermediate coarse mesh have been eliminated. Unlike the method discussed in Reference 13, the polynomial approximation of the FEM nodal deflections is now transfered directly to the final CFD mesh.

To summarize the current method, the transfer of deflections from the beam model to the CFD surface mesh starts with regions in which a continuous mathematical approximation of the nodal deflections is defined. Six subdomain regions are defined, as shown in Figure 6a. Up to sixth order polynomial functions can be defined in local optimally oriented coordinate systems, as shown in Figure 6b. The modal deflection, calculated in the local coordinate system, is 
transformed back into the global coordinate system. Finally, a merge/blend step is performed between the subdomains (see Figure 6c), which smoothly joins subdomains into a continuously deflected surface. Additional details regarding the smoothing process used for blending are found in Reference 13. An example of the final product is shown in Figure 6d.

\section{Results}

\section{A. Flutter Simulations with Linear Aerodynamics}

For the linear flutter analysis, the MSC.Nastran (SOL 145) $p-k$ method (with no looping) is used. A matched set of densities and velocities are calculated based on R134a as the test medium. Steady aerodynamic corrections based on CFD results are utilized to improve the fidelity of the aerodynamics used in the simulations. Figure 7 presents flutter onset results at zero degrees AoA for the v.19 and v.20 FEMs. The unloaded vibration modes and frequencies are used for these flutter analyses. Note that experimental LCO conditions at -1 and +1 degrees AoA are shown in Figures 7 and 8 for reference.

To perform flutter analyses with a time-accurate high-fidelity CFD code requires computations at a succession of dynamic pressures until instability is observed. To simplify the process of high-fidelity CFD computing at successive dynamic pressures, the flutter onset boundary was calculated in Reference 13 for unloaded conditions at zero degrees AoA. The v.19 FEM modes were used in that reference. The same approach is used here for conditions at zero degrees AoA using the v.20 FEM. To make a consistent comparison with these high-fidelity simulations, $p-k$ flutter calculations are also performed using unloaded v. 20 modes. A comparison of the flutter onset boundaries using the v.19 and v.20 FEMs can be seen in Figure 7. The model changes made in going from v.19 to v.20 have a significant effect on flutter onset. The fundamental flutter mechanism does not change, still being a coalescence of modes 3 ( $2^{\text {nd }}$ out-of-plane) and $4\left(1^{\text {st }}\right.$ torsion) at a frequency near $10 \mathrm{~Hz} .{ }^{13}$ However, the dynamic pressure at which flutter onset occurs is significantly higher using the v.20 as opposed to the v.19 FEM. The increase in flutter onset dynamic pressure can be accounted for by the larger difference in the $3^{\text {rd }}$ and $4^{\text {th }}$ mode frequencies in v.20, requiring a higher dynamic pressure at which the two modes to coallesce.

\section{B. High-Fidelity Aeroelastic Simulations}

Time-accurate high-fidelity aeroelastic simulations are performed with the Reynolds-averaged Navier-Stokes (RANS) code FUN3D. A study of the time step size, CFL number and number of sub-iterations necessary for accurate solutions was performed in a previous publication ${ }^{13}$ and is not repeated here. The time step used in the current LCO simulations is $2.36 \times 10^{-4}$ seconds. This results in 423 time steps per $10 \mathrm{~Hz}$ cycle. The flow field CFL number used is 75 , and the turbulence CFL number is 50, with 20 subiterations per time step. Subiterative convergence of the lift and moment coefficient has been previously studied, ${ }^{13}$ and shown to converge well in 18-20 subiterations. Note that at this time, a spatial grid convergence study has not been conducted.

The computing procedure is that steady state rigid solutions are obtained, then static aeroelastic solutions are converged with an artificially high structural damping value. The time-accurate high-fidelity aeroelastic solution, with the measured structural damping ratio of one percent, is commenced by giving all 16 modes an initial modal velocity. The solutions are continued until a steady state LCO amplitude is reached, or else the LCO amplitude is trending toward zero. In order to estimate the LCO onset boundary for zero degree AoA conditions, time-accurate RANS solutions have been computed at dynamic pressure intervals of 10 to 15 psf. Figure 7 presents the LCO onset boundaries for the v.19 (solid red) and v.20 (solid black) FEMs as they are estimated from these unsteady RANS solutions.

There is a significant difference in LCO onset between the v. 19 and v.20 FEMs. Besides the general increase in the dynamic pressure at LCO onset using the v.20 FEM, there is a more profound difference in the LCO onset boundaries above Mach 0.80. With v.20, no sustained LCO was found at Mach 0.82 until a dynamic pressure of $110 \mathrm{psf}$. The estimated LCO onset line for v.20 and the conditions with LCO and without LCO (closed/open delta) in Figure 7 are at zero degrees AoA. Although not shown, the lack of sustained LCO at Mach 0.82 below 100-110 psf was also seen in v. 20 simulations at -1 and +1 degrees AoA. Thus, a sharp rise in flutter/LCO onset at all AoAs occurs between Mach 0.78 and 0.82 . This is a very different behavior than was computed with FEM v.19.

Coggin et al. ${ }^{10}$ performed flutter analyses using linear aerodynamics and a geometrically nonlinear structural model at -1 and +1 degree AoA. One of their findings is that the MSC.Nastran flutter analysis, corrected with CFD data, did not exhibit flutter beyond Mach $0.82 .{ }^{10}$ The CFD generated aerodynamic weighting factors were seen as a key factor in the lack of flutter beyond Mach 0.82. At this Mach number, an aft shift in the location of the center of 
pressure, caused by a shift in shock location, is the reason given for no flutter instability. Contrary to this result, the time-accurate RANS analysis in Reference 13, using the SUGAR FEM v.19, found LCO onset at fairly low dynamic pressures beyond Mach 0.82 (see the solid red line in Figure 7). The current LCO onset using v.20 is much more consistent with the flutter onset presented by Coggin et al. ${ }^{10}$ It also confirms another finding of Coggin et al., that the flutter/LCO onset is very sensitive to the mass and/or stiffness distribution of the structure.

The flutter onset calculations published in Reference 10 also indicated an AoA dependence. The present flutter instability boundaries at -1 and +1 degree AoA computed with the $p-k$ method, shown in Figure 8 , also show a sensitivity to AoA. The flutter onset boundary at a positive AoA is at a higher dynamic pressure than that a negative AoA. This can largely be accounted for by the fact that the positive wing loading at +1 degree results in higher frequencies in the first two out-of-plane bending modes, in particular mode 3 (See Table 1). Negative wing loading increases the frequency of the out-of-plane bending modes, but not to the extent that positive loading does, thus accounting for the difference in flutter onset dynamic pressure at -1 and +1 degree AoA. It also accounts for the fact that the flutter onset boundaries at -1 and +1 degree AoA are at higher dynamic pressures than that for zero degrees AoA.

In the present paper, time-accurate high-fidelity RANS simulations at -1 and +1 degrees AoA have been performed at five flow conditions of Mach number and dynamic pressure. These conditions are shown in Figure 8. Three conditions are near flutter onset (Mach 0.70, 0.75 and 0.78 ) while two conditions are at much higher dynamic pressures (100 psf at Mach 0.70 and 0.75). These conditions enable assessment of the influence of dynamic pressure and Mach number on the LCO amplitudes and frequencies. Figures 9-13 present results from these simulations. Note that these dynamic pressures are not at LCO onset. Rather by their sustained LCO amplitudes or oscillatory damping, the simulations provide an indication of how close the conditions are to LCO onset. Furthermore, the conditions at Mach 0.70 and 0.75 and $100 \mathrm{psf}$ provide a way to assess purely Mach number effects.

Turning attention to Figure 9a, results are shown for a specific wing tip location, near the leading edge, where an accelerometer was located during the TDT test. The location of the forward wing tip accelerometer is shown in Figure 10. The RANS LCO amplitudes at AoAs of -1 and +1 degree are smaller than at 0 degree in all cases, except Mach 0.78 and 75 psf. The larger amplitude at 0 degree AoA is consistent with the MSC.Nastran results in that flutter onset at 0 degrees is at a lower dynamic pressure than the flutter onset at AoAs of -1 and +1 degrees. For example, compare Figure 7 with 8 . Therefore, at a given dynamic pressure above flutter onset, the 0 degree solution will be deeper into flutter. Note that $30 \mathrm{~g}$ 's is roughly equivalent to \pm 3 inches wing tip deflection.

Note also that the RANS LCO amplitude is larger at AoA of -1 than at +1 degree. This is also consistent with the present MSC.Nastran solutions, and those of Coggin et al, ${ }^{10}$ which show flutter onset at -1 degree at a lower dynamic pressure than at +1 degree. Note however, that the LCO amplitude difference due to AoA is somewhat smaller at dynamic pressures near LCO onset. This can be seen best in Figure 9b. Observing the damped oscillations in Figure 13 at Mach 0.78 and 75 psf, suggests this dependence of LCO onset on AoA may not hold true at that Mach number. Finally it can be noted that the LCO amplitudes at $-1,0$ and +1 degrees AoA are smaller overall at Mach 0.75 than at 0.70 . These features provide evidence that compressibility, or transonic effects associated with mean shock location, have a significant influence on the LCO/flutter onset behavior in general, and dependence on AoA in particular.

LCO frequencies also show a slight AoA dependence. Figure 9c presents high-fidelity simulation frequencies while Figure 11 presents flutter frequencies computed with the $p-k$ method. With all methods the LCO (or flutter) at +1 degree AoA has slightly higher frequency than -1 degree. The CFD based LCO frequencies (using the v.20 FEM) range between 10.5 and $11.0 \mathrm{~Hz}$. The MSC.Nastran flutter frequencies, using v.20 FEM, range between 9.7 and 10.2 Hz. As expected, using the v.19 SUGAR FEM, the flutter frequencies computed by the $p-k$ method are somewhat higher than the v.20 FEM flutter frequencies.

Figures 12-13 present calculated time histories of the forward wing tip accelerations in g's. Time histories are presented at all three AoAs for a dynamic pressure near LCO onset. Mach 0.75 and 0 degree AoA has an amplitude of approximately $3 \mathrm{~g}$ 's. The amplitudes of the oscillations at -1 and +1 degree AoA are trending toward zero. The oscillations at +1 degree AoA appear to be somewhat more damped than at -1 degree. This is consistent with the AoA dependence discussed earlier. Figure 13 presents the calculated time history response of the forward wing tip acceleration at Mach 0.78 and 75 psf. Note, for this case, the oscillations at +1 degree appear to be somewhat less damped than those oscillations at -1 and 0 degrees AoA. 


\section{Conclusions}

This paper has focused on the simulation of the flutter and limit cycle oscillation of the Boeing Subsonic Ultra Green Aircraft Research truss-braced wing wind-tunnel model. Analyses have been performed using unloaded vibration modes for conditions at zero AoA and vibration modes about nonlinear static aeroelastic solutions for conditions at -1 and +1 degree AoA. Flutter simulations have been performed using linear and nonlinear Navier-Stokes aerodynamics. The analyses at zero AoA using the post-test finite element model (v.20) is found to predict flutter onset generally closer to the wind-tunnel test data for limit cycle oscillation onset than does the pre-test finite element model (v.19). The high-fidelity CFD simulations using the v.20 FEM indicate a sharp rise in LCO onset dynamic pressure between Mach 0.78 and 0.82. This is much different than the previous (pre-test) results using the v.19 FEM. This result indicates that LCO or flutter onset is quite sensitive to the mass and/or stiffness distribution of the wing. The result also substantiates similar findings by previous researchers. This and previous studies of TBW aircraft have found flutter onset to be a function of AoA. In simulations with linear aerodynamics, flutter onset occurs earlier at -1 degree than at +1 degree AoA. The Navier-Stokes aeroelastic simulations, while not sufficiently detailed to assess the precise LCO onset, do show LCO amplitude dependence on AoA. At conditions well beyond LCO onset, -1 degree AoA responses exhibit larger amplitude LCO than do responses at +1 degree, for the same dynamic pressure. The high-fidelity simulations also show larger LCO amplitude at zero AoA than at either -1 or +1 degree. This is consistent with the fact that linear aerodynamics puts flutter onset at zero degrees AoA at a much lower dynamic pressure than at -1 or +1 degree. The somewhat unexpected AoA dependence at Mach 0.78 and 75 psf requires further exploration, perhaps with dynamic pressures just above LCO onset. This and the generally lower LCO amplitudes at Mach 0.75 than 0.70 provide evidence that compressibility or transonic effects and mean shock location in particular, have a significant influence on the LCO/flutter onset. It is clear from this and previous studies that at least static, if not dynamic, aeroelastic analyses with nonlinear structure and nonlinear aerodynamic effects (at least through correction factors) are necessary to adequately predict the behavior of the TBW vehicle.

\section{References}

\footnotetext{
${ }^{1}$ Kenway, G. K. W. and Martins, J. R., "Multi-Point High-Fidelity Aerostructural Optimization of a Transport Aircraft Configuration," Journal of Aircraft, Vol. 51, No. 1, January 2014, pp. 144-160.

${ }^{2}$ Hwang, J. T., Kenway, G. K. W., and Martins, J. R., "Geometry and Structural Modeling for High-Fidelity Aircraft Conceptual Design Optimization," 15th AIAA/ISSMO Multidisciplinary Analysis and Optimization Conference, No. AIAA-2014-2041, AIAA, Atlanta, Georgia, June 2014.

${ }^{3}$ Gundlach, J. F., Tetrault, P. A., Gern, F. H., Naghshineh-Pour, A. H., Schetz, J. A., Mason, W. H., Kapania, R. K., Grossman, B., and Haftka, R. T., "Conceptual Design of Strut-Braced wing Transonic Transport," Journal of Aircraft, Vol. 37, No. 6, Nov-Dec 2000, pp. 976-983.

${ }^{4}$ Gur, O., Bhatia, M., Schetz, J. A., Mason, W. H., Kapania, R. K., and Mavris, D. H., "Design Optimization of a Truss-Braced-Wing Transonic Transport Aircraft," Journal of Aircraft, Vol. 47, No. 6, 2010, pp. 1907-1917.

${ }^{5}$ Meadows, N. A., Schetz, J. A., Kapania, R. K., and Bhatia, M., "Multidisciplinary Design Optimization of Medium-Range Transonic Truss-Braced Wing Transport Aircraft," Journal of Aircraft, Vol. 49, No. 6, 2012, pp. 1025-1035.

${ }^{6}$ Bhatia, M., Kapania, R. K., and Haftka, R. T., "Structural and Aeroelastic Characteristics of Truss-Braced Wings: A Parametric Study," Journal of Aircraft, Vol. 49, No. 1, January-February 2012, pp. 302-310.

${ }^{7}$ Mallik, W., Kapania, J. A., and Schetz, J. A., "Multidisciplinary Design Optimization of Medium-Range Transonic Truss-Braced Wing Aircraft with Flutter Constraint," 54th AIAA/ASME/ASCE/AHS/ASC Structures, Structural Dynamics, and Materials Conference, No. AIAA 20131454, AIAA, Boston, Massachusetts, April 2013.

${ }^{8}$ Gupta, R., Mallik, W., Kapania, J. A., and Schetz, J. A., "Multidisciplinary Design Optimization of Subsonic Truss-Braced Wing Cargo Aircraft," 52nd Aerospace Sciences Meeting, No. AIAA 2014-0186, National Harbor, Maryland, January 2014.

${ }^{9}$ Nam, T., Chakraborty, I., Gross, J., Mavris, D. H., Schetz, J. A., and Kapania, J. A., "Multidisciplinary Design Optimization of a TrussBraced Wing Concept," 14th AIAA Aviation Technology, Integration, and Operations Conference, No. AIAA 2014-2423, Atlanta, GA, June 2014.

${ }^{10}$ Coggin, J. M., Kapania, R. K., Zhao, W., Schetz, J. A., Hodigere-Siddaramaiah, V., Allen, T. J., and Sexton, B. W., "Nonlinear Aeroelastic Analysis of a Truss Braced Wing Aircraft," 55th AIAA/ASMe/ASCE/AHS/SC Structures, Structural Dynamics, and Materials Conference, No. AIAA-2014-0335, National Harbor, MD, Jan. 2014.

${ }^{11}$ Bradley, M. K. and Droney, C. K., "Subsonic Ultra Green Aircraft Research: Truss Braced Wing Design Exploration," Contractor report, The Boeing Company, June 2014.

${ }^{12}$ Bradley, M. K., Droney, C. K., and Allen, T. J., "Subsonic Ultra Green Aircraft Research: Truss Braced Wing Aeroelastic Test Report," Contractor report, The Boeing Company, June 2014.

${ }^{13}$ Bartels, R. E., Scott, R. C., Funk, C. J., Allen, T. J., and Sexton, B. W., "Computed and Experimental Flutter/LCO Onset for the Boeing Truss-Braced Wing Wind-Tunnel Model,” 32st AIAA Applied Aerodynamics Conference, No. AIAA-2014-9999, Atlanta, GA, June 2014.

${ }^{14}$ NASA LaRC, Hampton, VA, FUN3D Manual, Nov. 2008.

${ }^{15}$ Parlette, E. and Garriz, J., VGRID: Unstructured Grid Generation Program Version 3.9 User's Guide, ViGYAN, Inc., Hampton, VA, 2007.
} 
Table 1. Variation of modal frequencies with flow condition, unloaded and $100 \mathrm{psf}$ at Mach 0.75.

\begin{tabular}{ccccc}
\hline \hline Mode No & Description & Freq., unloaded (Hz) & Freq., AoA -1 deg (Hz) & Freq., AoA +1 deg (Hz) \\
\hline 1 & $1^{\text {st }}$ out-of-plane wing bending & 5.04 & 5.07 & 5.17 \\
2 & $1^{s t}$ in-plane wing bending & 5.70 & 5.60 & 5.58 \\
3 & $2^{\text {nd }}$ out-of-plane wing bending & 8.44 & 8.50 & 8.60 \\
4 & $1^{\text {st }}$ wing/nacelle torsion & 11.3 & 11.2 & 11.3 \\
5 & $3^{\text {rd }}$ out-of-plane wing bending & 18.5 & 18.0 & 18.4 \\
\end{tabular}




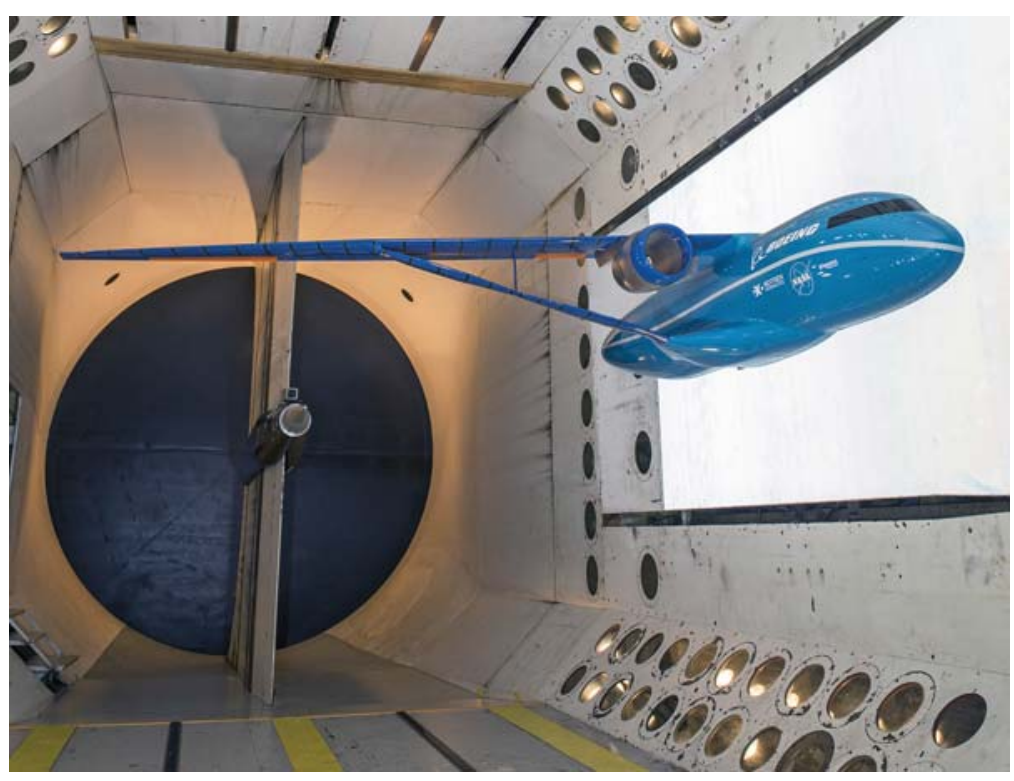

Figure 1. TDT SUGAR model.

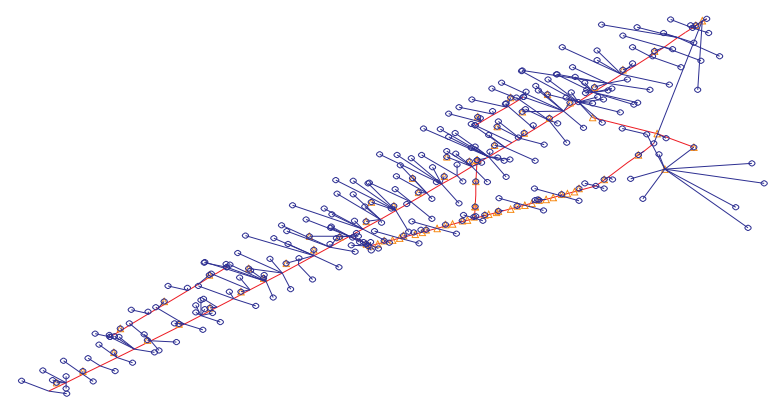

(a) Boeing MSC.Nastran FEM of wind-tunnel model.

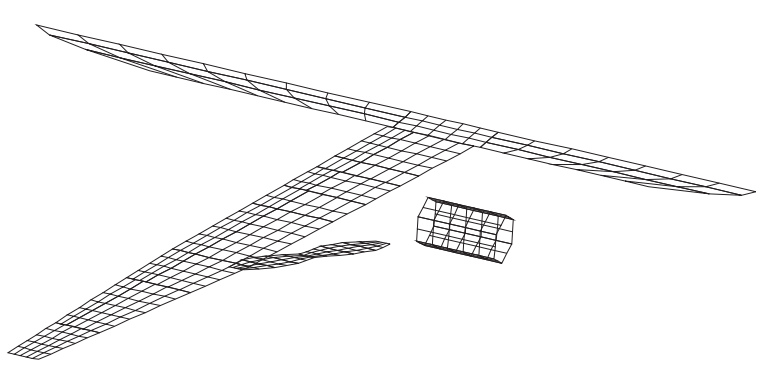

(b) Aero boxes used in linear flutter analyses.

Figure 2. Finite element structural and aerodynamic models. 


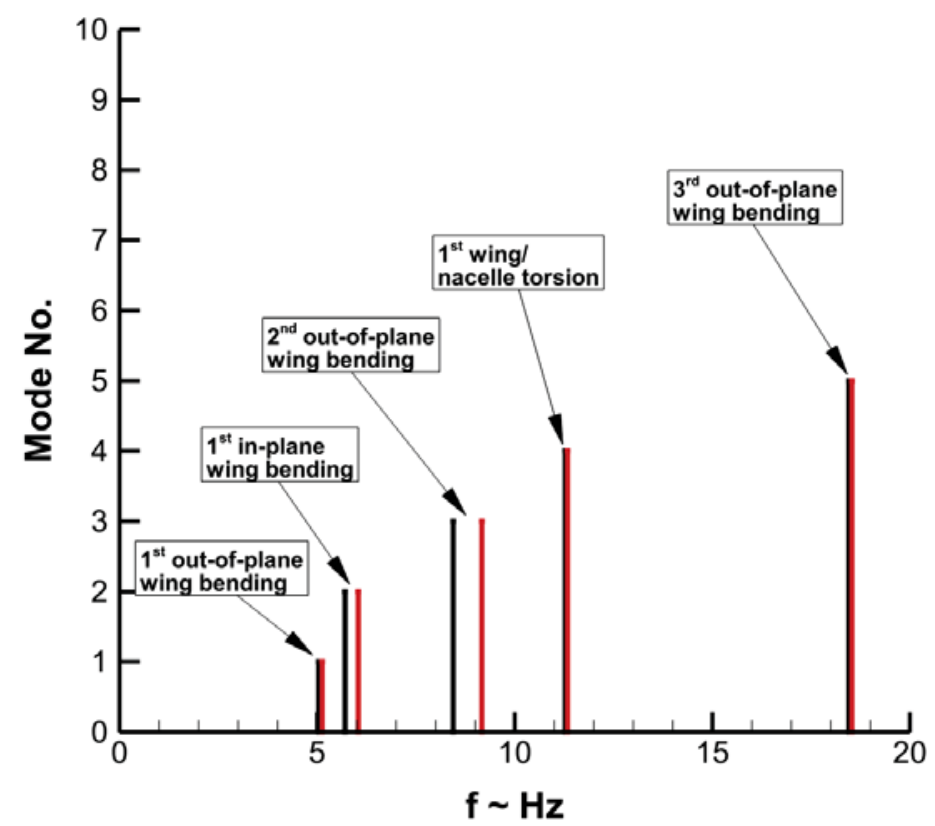

Figure 3. Comparison of modal frequencies for v.19 and v.20 (red - v.19, black - v.20).

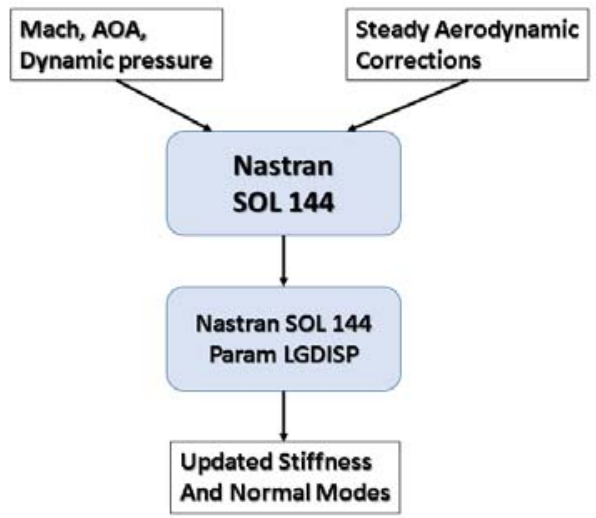

Figure 4. Nastran process for nonlinear static solution. 


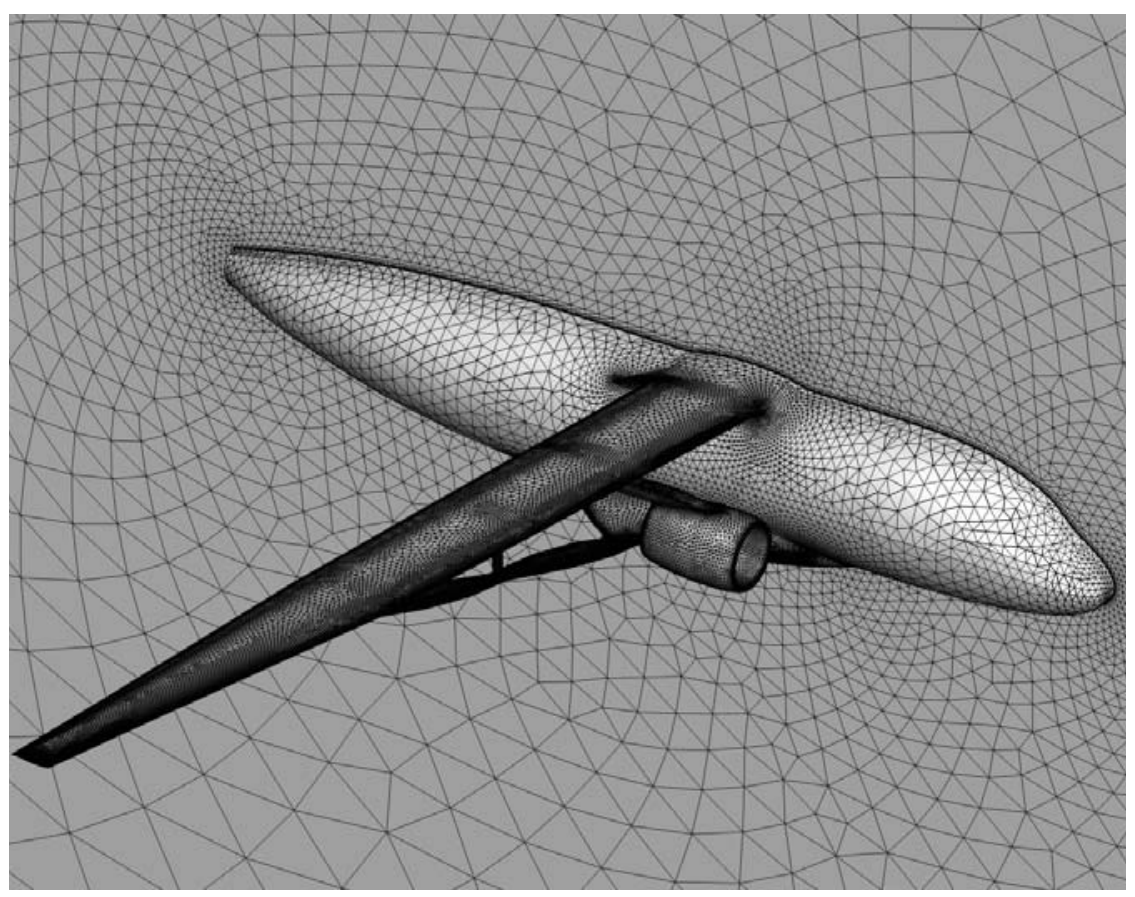

Figure 5. CFD surface mesh. 


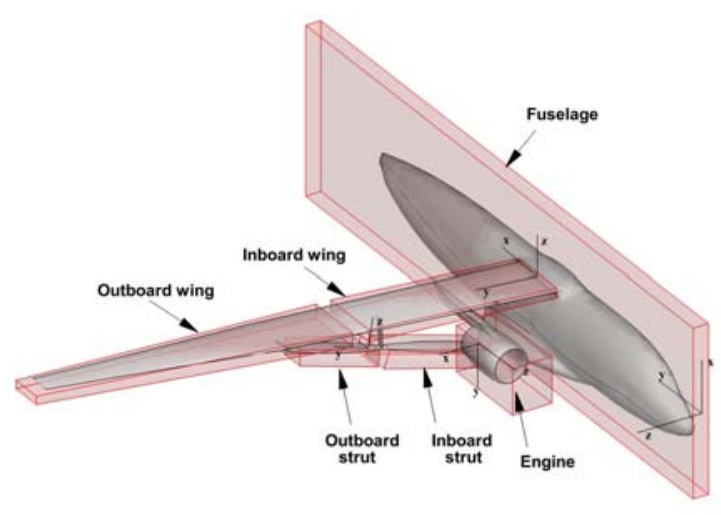

(a) TBW subdomains for mode generation.

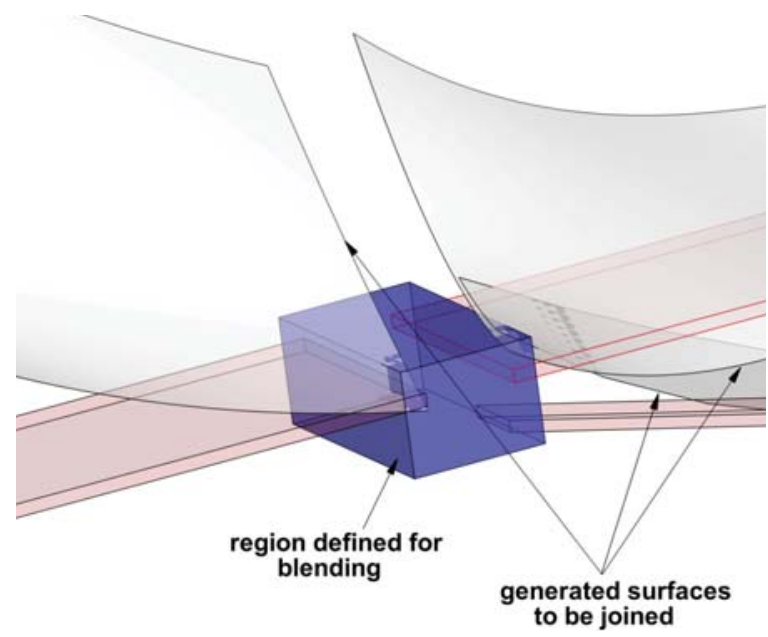

(c) Blending region.

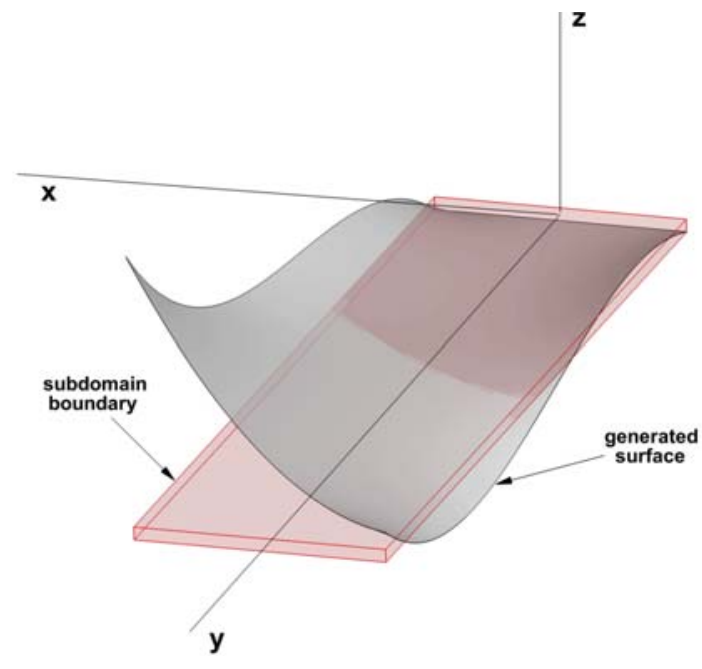

(b) Subdomain definitions.

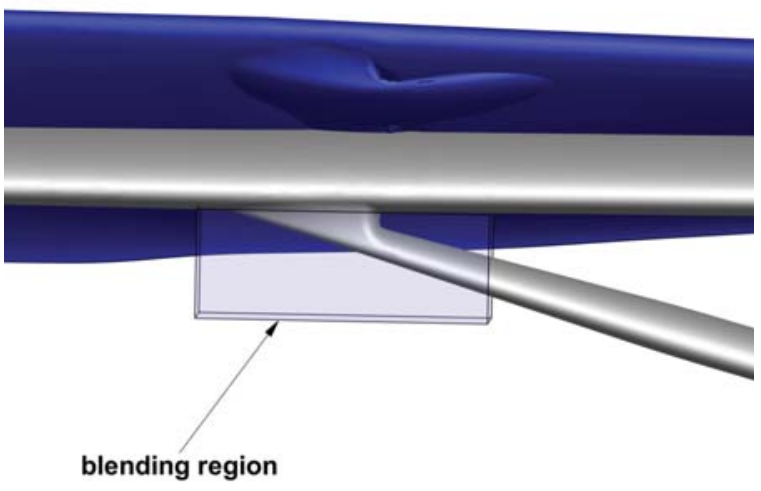

(d) Initial (gray)/final (blue) surfaces.

Figure 6. Beam model deflection transfer to CFD surface. 


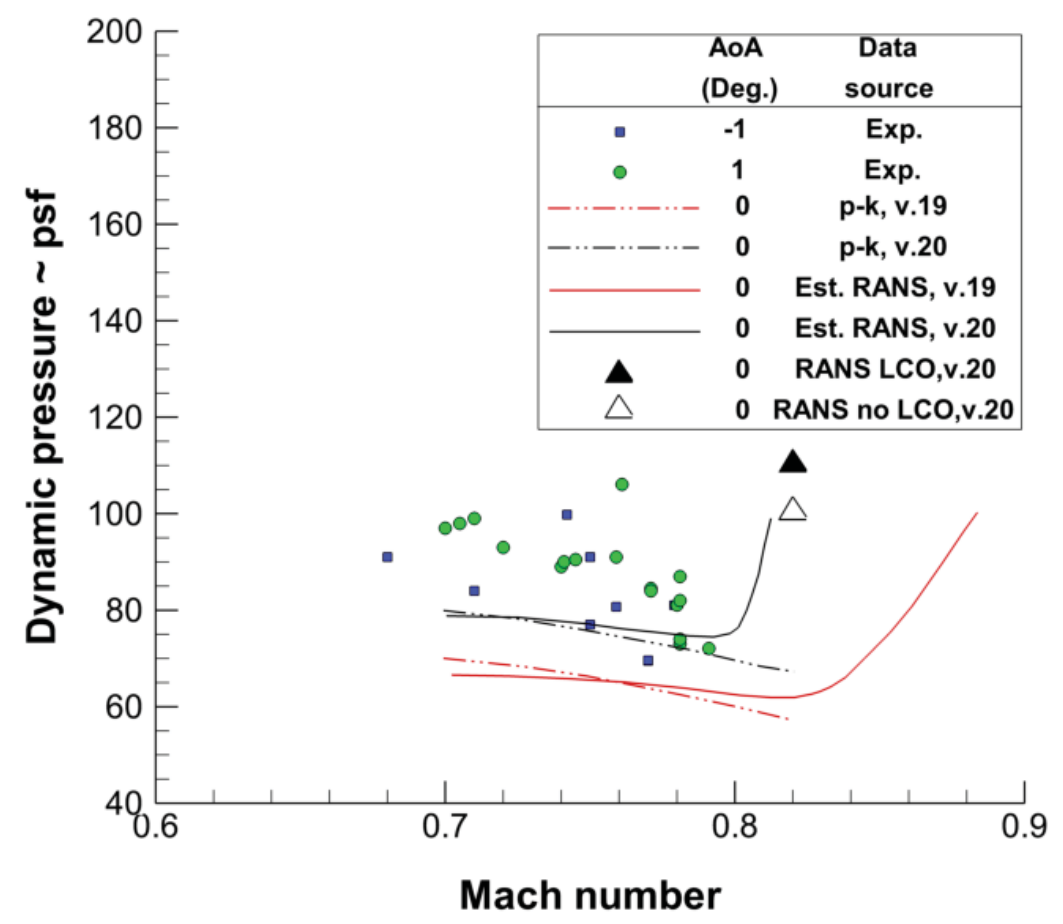

Figure 7. LCO and linear flutter onset.

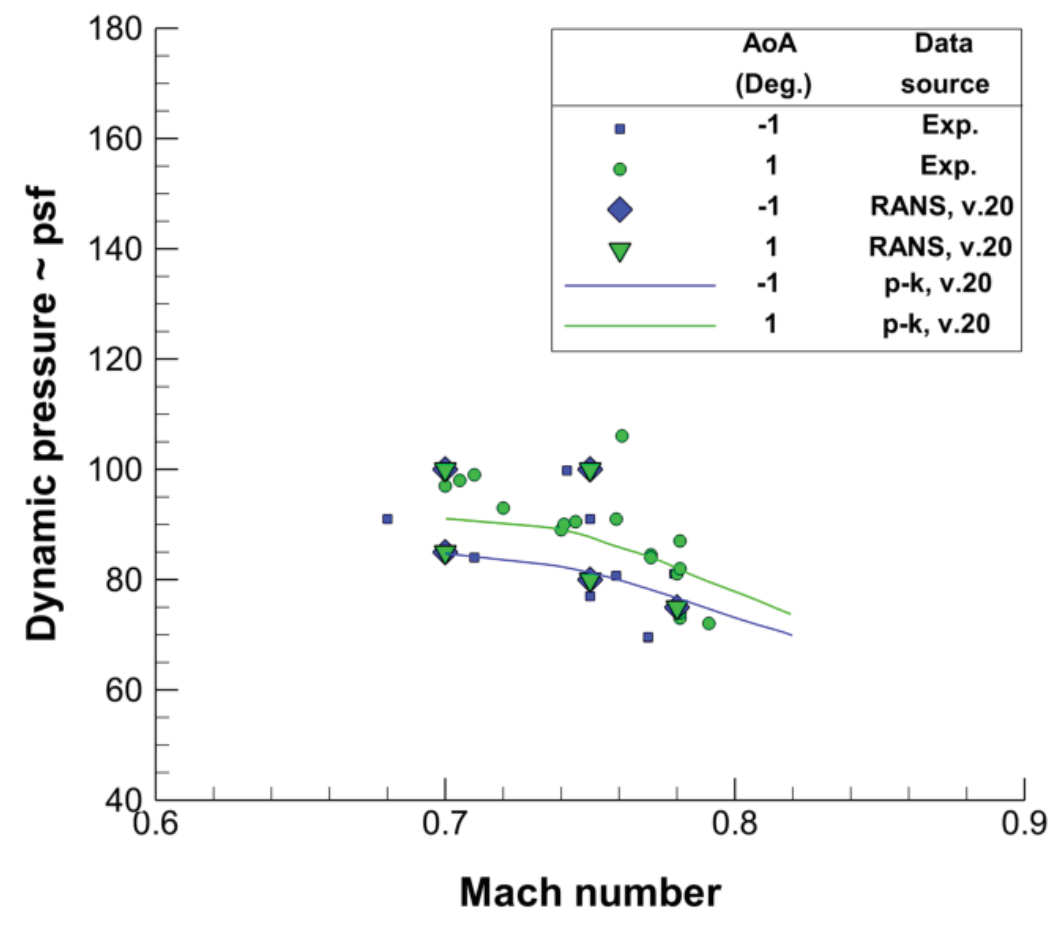

Figure 8. LCO conditions and linear flutter onset. 


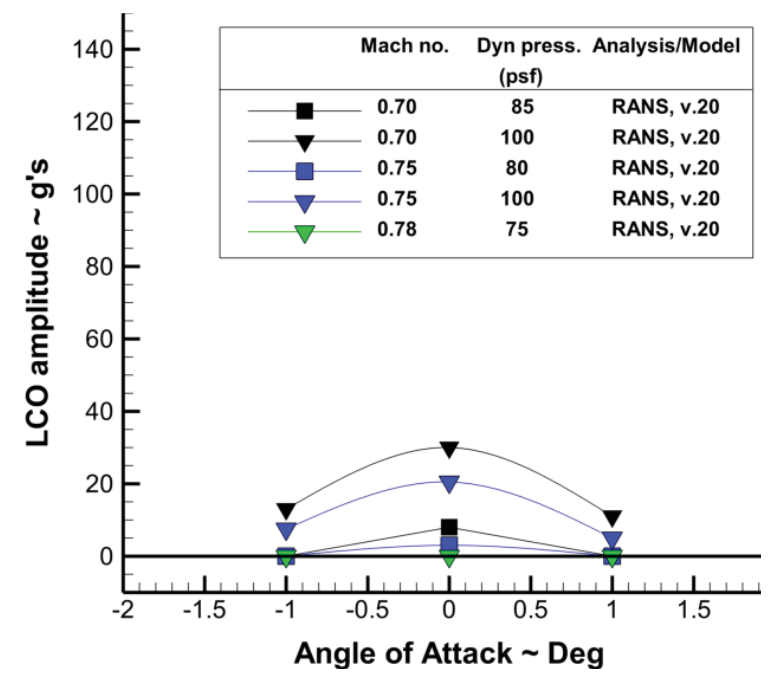

(a) Amplitude.

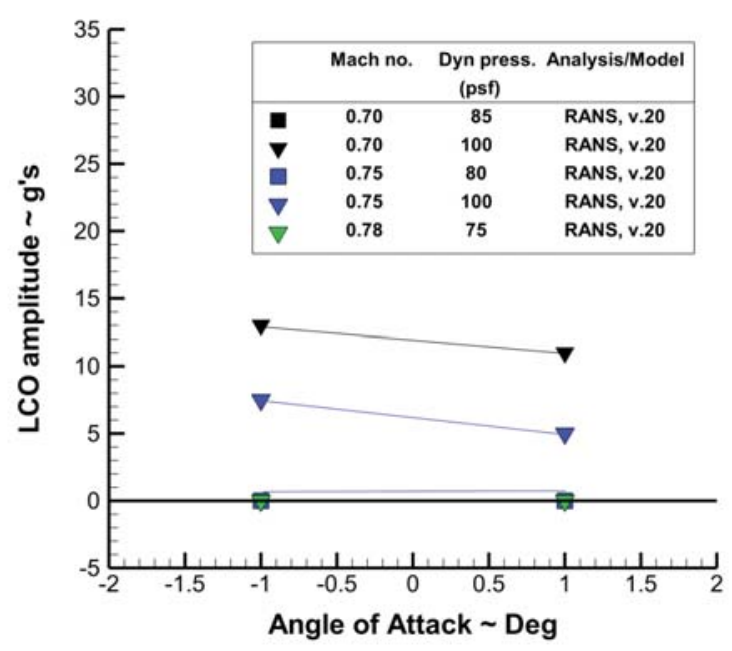

(b) Amplitude at AOA -1/+1 degree.

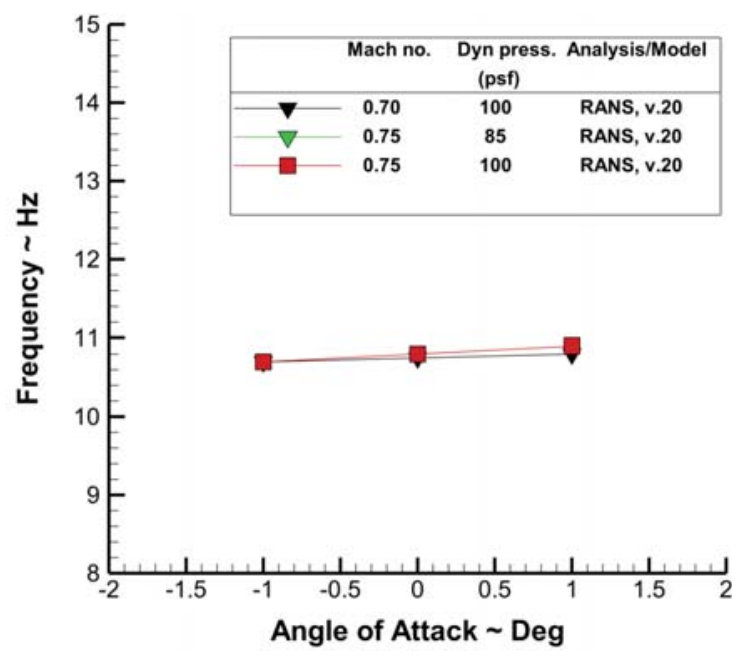

(c) Frequency.

Figure 9. LCO (Computed), v.20 FEM. 


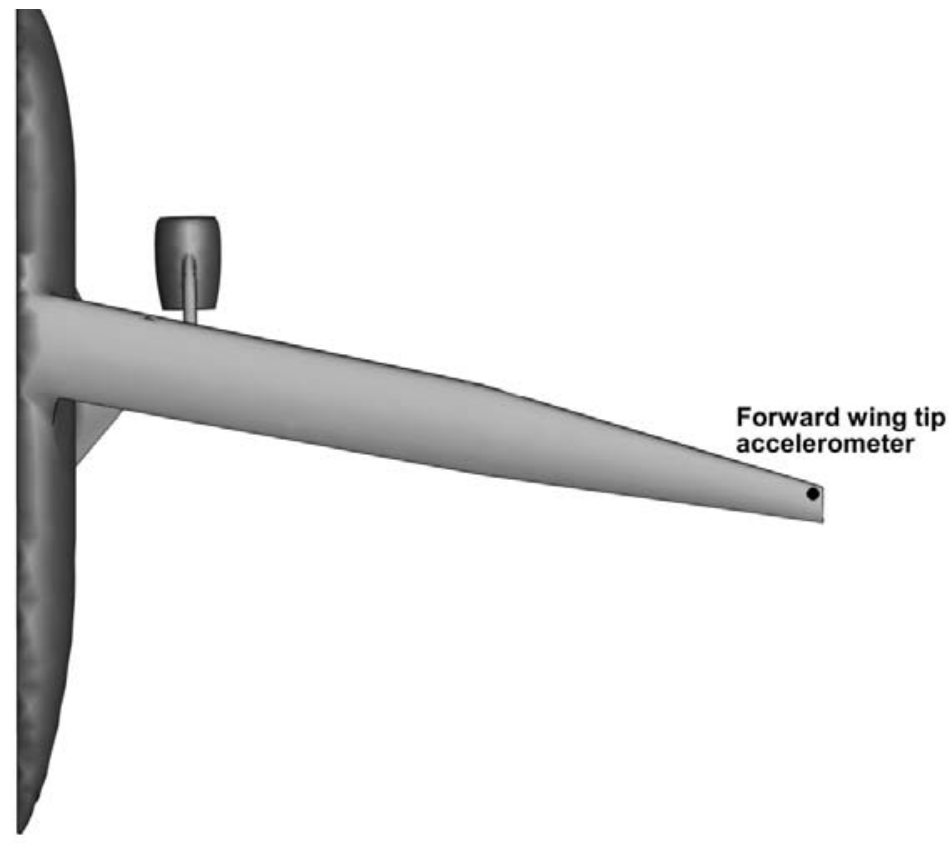

Figure 10. Location of the forward wing tip accelerometer.

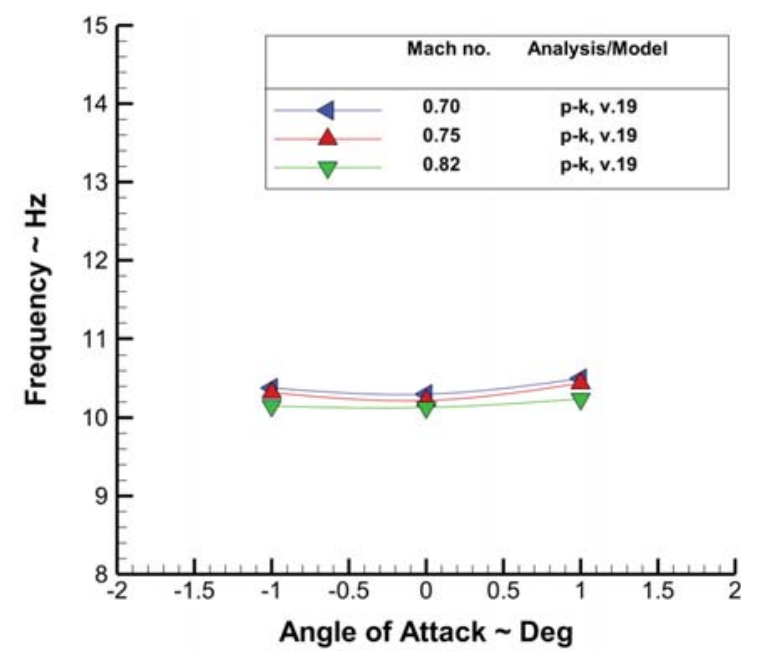

(a) v. 19 .

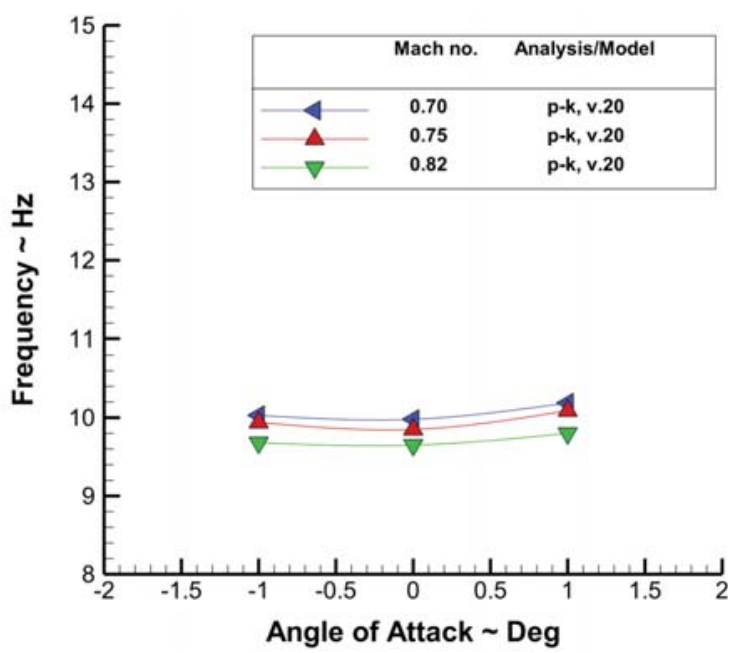

(b) v. 20 .

Figure 11. $p-k$ flutter frequencies. 


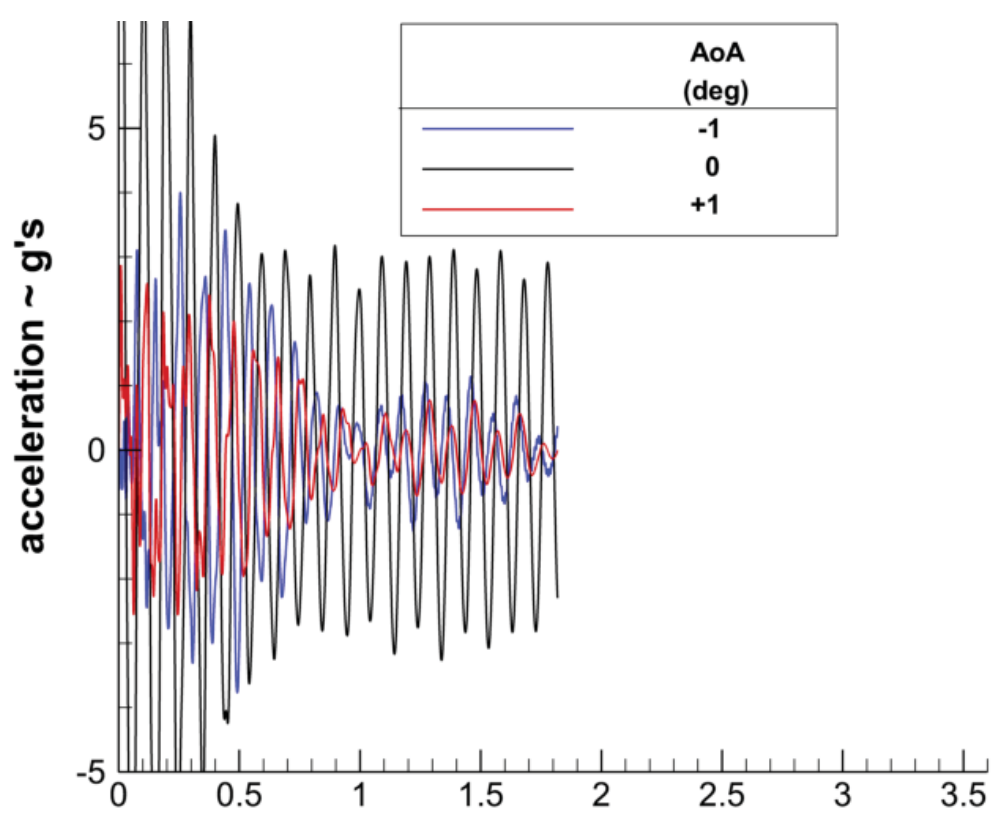

Figure 12. RANS simulation, Mach 0.75 , dynamic pressure 80 psf.

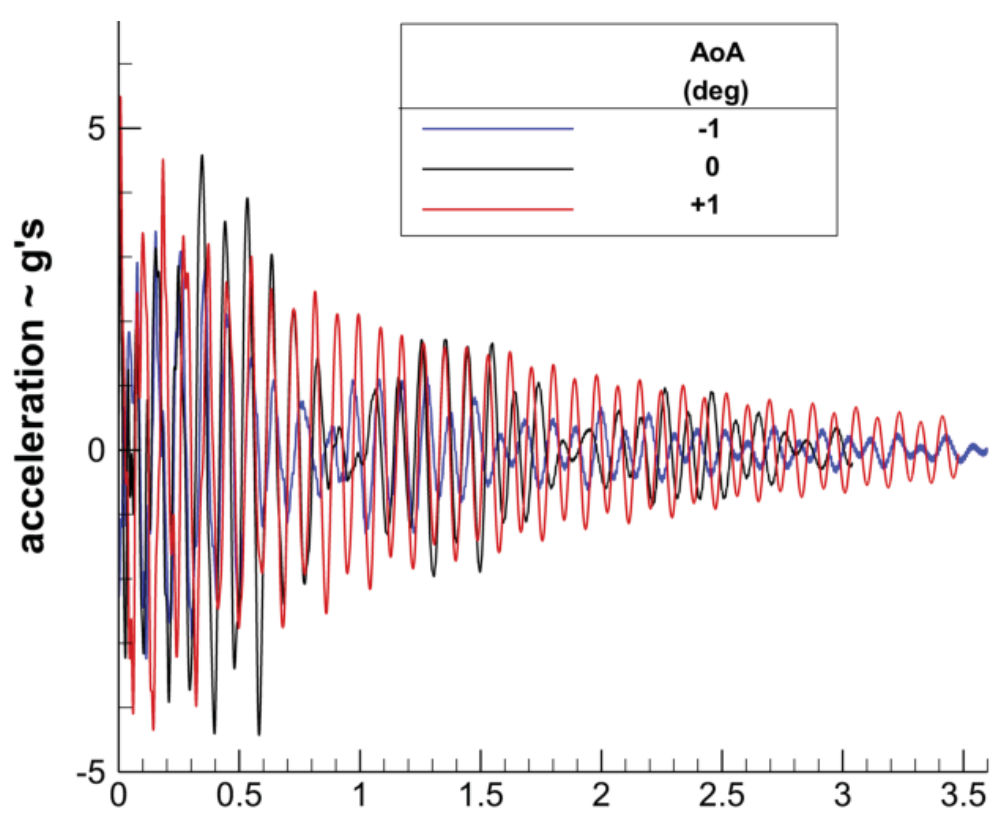

Figure 13. RANS simulation, Mach 0.78, dynamic pressure 75 psf. 